\title{
Population inversion in a magnetized hydrogen plasma expansion as a consequence of the molecular mutual neutralization process
}

\author{
Citation for published version (APA): \\ Harskamp, van, W. E. N., Brouwer, C. M., Schram, D. C., \& Engeln, R. A. H. (2011). Population inversion in a \\ magnetized hydrogen plasma expansion as a consequence of the molecular mutual neutralization process. \\ Physical Review E - Statistical, Nonlinear, and Soft Matter Physics, 83(3), 036412-1/9. [036412]. \\ https://doi.org/10.1103/PhysRevE.83.036412
}

DOI:

10.1103/PhysRevE.83.036412

Document status and date:

Published: 01/01/2011

\section{Document Version:}

Publisher's PDF, also known as Version of Record (includes final page, issue and volume numbers)

\section{Please check the document version of this publication:}

- A submitted manuscript is the version of the article upon submission and before peer-review. There can be important differences between the submitted version and the official published version of record. People interested in the research are advised to contact the author for the final version of the publication, or visit the DOI to the publisher's website.

- The final author version and the galley proof are versions of the publication after peer review.

- The final published version features the final layout of the paper including the volume, issue and page numbers.

Link to publication

\footnotetext{
General rights

- You may freely distribute the URL identifying the publication in the public portal. follow below link for the End User Agreement:

www.tue.nl/taverne

\section{Take down policy}

If you believe that this document breaches copyright please contact us at:

openaccess@tue.nl

providing details and we will investigate your claim.
}

Copyright and moral rights for the publications made accessible in the public portal are retained by the authors and/or other copyright owners and it is a condition of accessing publications that users recognise and abide by the legal requirements associated with these rights.

- Users may download and print one copy of any publication from the public portal for the purpose of private study or research.

- You may not further distribute the material or use it for any profit-making activity or commercial gain

If the publication is distributed under the terms of Article $25 \mathrm{fa}$ of the Dutch Copyright Act, indicated by the "Taverne" license above, please 


\title{
Population inversion in a magnetized hydrogen plasma expansion as a consequence of the molecular mutual neutralization process
}

\author{
W. E. N. van Harskamp, C. M. Brouwer, D. C. Schram, M. C. M. van de Sanden, and R. Engeln* \\ Department of Applied Physics, Eindhoven University of Technology, \\ P.O. Box 513, NL-5600 MB Eindhoven, The Netherlands \\ (Received 5 October 2010; published 29 March 2011)
}

\begin{abstract}
A weakly magnetized expanding hydrogen plasma, created by a cascaded arc, was investigated using optical emission spectroscopy. The emission of the expanding plasma is dominated by $\mathrm{H}_{\alpha}$ emission in the first part of the plasma expansion, after which a sharp transition to a blue afterglow is observed. The position of this sharp transition along the expansion axis depends on the magnetic field strength. The blue afterglow emission is associated with population inversion of the electronically excited atomic hydrogen states $n=4-6$ with respect to $n=3$. By comparing the measured densities with the densities using an atomic collisional radiative model, we conclude that atomic recombination processes cannot account for the large population densities observed. Therefore, molecular processes must be important for the formation of excited states and for the occurrence of population inversion. This is further corroborated at the transition from red to blue, where a hollow profile of the excited states $n=4-6$ in the radial direction is observed. This hollow profile is explained by the molecular mutual neutralization process of $\mathrm{H}_{2}{ }^{+}$with $\mathrm{H}^{-}$, which has a maximum production for excited atomic hydrogen $1-2 \mathrm{~cm}$ outside the plasma center.
\end{abstract}

DOI: 10.1103/PhysRevE.83.036412

PACS number(s): $52.25 . \mathrm{Xz}, 52.70 . \mathrm{Kz}, 34.50 . \mathrm{Ez}$

\section{INTRODUCTION}

The development of efficient sources of reactive hydrogen radicals is important in many research fields and applications. For instance, atomic hydrogen radicals serve as primary reactive particles for surface modification or thin-film deposition [1,2]. For fusion plasma heating, one of the main research challenges is to develop efficient negative ion sources. A promising route is via dissociative attachment of rovibrationally excited hydrogen molecules $\mathrm{H}_{2}{ }^{\mathrm{rv}}$ [3]. Studying the kinetics of $\mathrm{H}_{2}{ }^{\mathrm{rv}}$ is of key importance in understanding negative ion formation processes in both volume and surface reactions [4]. The ro-vibrationally excited molecules are an important precursor in volume reaction produced molecular activated recombination (MAR) processes [5-7]. In this paper the importance of several MAR processes is examined, and the formation of excited atom production via the mutual neutralization process of $\mathrm{H}^{-}$and $\mathrm{H}_{2}{ }^{+}$is discussed in detail [8-11].

A wall-stabilized cascaded arc attached to a low-pressure vessel has been shown to be a reliable source for the production of well-defined fluxes of atomic and molecular radicals (see Fig. 1). This kind of plasma source was first introduced by Maecker [12] as a light source using argon as the carrier gas. The study of expanding argon plasmas was continued by Kroesen et al. [13] as a particle source in a deposition chamber. This method of deposition was shown to be efficient and fast for growing hydrogenated amorphous carbon films $(\mathrm{a}-\mathrm{C}: \mathrm{H})$. The fundamental aspects of argon plasmas expanding from a cascaded arc were studied extensively by van de Sanden et al. [14,15]. Work on hydrogen as a carrier gas was first done by de Graaf et al. [5] and Meulenbroeks et al. [16] by looking at the dynamics and kinetics of nonmagnetized expanding hydrogen plasmas at a pressure of

*r.engeln@tue.nl
$100 \mathrm{~Pa}$. It was demonstrated that in the hydrogen expansion an anomalously high recombination occurs, which leads to a very effective destruction of ions and electrons. To avoid this fast recombination, an external magnetic field and low background pressure were used to produce a hydrogen plasma expansion with two distinct regions. This separation is observed as a sharp transition from a red plasma to a blue afterglow, as reported in [17-19] (see Fig. 2). Akatsuka et al. [20] measured, with optical emission spectroscopy (OES), the occurrence of population inversion in the downstream plasma. The population mechanism in the blue afterglow is generally believed to be three-body recombination, as recently reported in Ref. [21] using a Radiation frequency (RF) plasma. However, Qing et al. [22] concluded that three-body recombination cannot be responsible for the observed population inversion in the magnetized hydrogen expansion because of the too low electron density and too high electron temperature [23]. Both parameters were obtained with a Langmuir double probe. Qing et al. showed the importance of molecular processes and proposed molecular mutual neutralization of $\mathrm{H}_{2}{ }^{+}+\mathrm{H}^{-}$as a mechanism to produce highly excited atomic hydrogen. A multicrossing Landau-Zener model was applied to calculate the molecular mutual neutralization cross section as a function of the quantum states $n$ [24]. The calculated cross section is rather large and has a maximum efficiency of excitation in the outgoing channels for the states $n=4,5$, and 6 . The importance of this reaction was further explored in Ref. [19], where the production rate of each excited state in the blue afterglow correlates very well with the shape of the cross section of the molecular mutual neutralization process.

In this paper we report on the production of excited $\mathrm{H}$ atoms in a magnetically confined hydrogen expansion. An important precursor for the formation of excited $\mathrm{H}$ atoms is $\mathrm{H}_{2}{ }^{\mathrm{rv}}$, which is produced via a wall association process at the anode nozzle of the arc and the reactor vessel [7]. These $\mathrm{H}_{2}{ }^{\mathrm{rv}}$ molecules flow into the plasma locally, producing $\mathrm{H}^{-}$ions via the dissociative attachment process [25]. The 


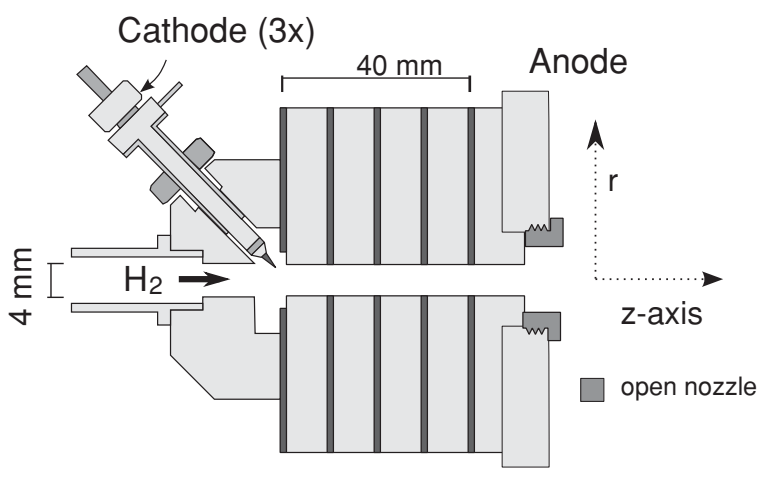

FIG. 1. The plasma under investigation is generated from flowing hydrogen using a wall-stabilized cascaded arc. Inside the channel the pressure is $10^{4} \mathrm{~Pa}$ and the temperature is $1-1.5 \mathrm{eV}$.

$\mathrm{H}^{-}$ions recombine with $\mathrm{H}_{2}{ }^{+}$to form hydrogen atoms in highly excited states, causing population inversion. This work continues the discussion of MAR processes in a cascaded arc [19,22]. New evidence for the existence of MAR processes is given by examining atomic state distributions and using a collisional radiative model. These results show the promising possibility of using a wall-stabilized cascaded arc as a $\mathrm{H}^{-}$ion source.

\section{EXPERIMENTAL SETUP AND DIAGNOSTICS}

\section{A. Plasma source and expansion}

The cascaded arc that serves as the plasma source for the experiments consists of three tungsten-lanthanum cathodes, four water-cooled copper plates that are separately insulated by PVC spacers and vacuum sealed by rubber O-rings, and a copper anode end-plate (see Fig. 1). The hydrogen gas flows with $3000 \mathrm{sccm}\left(1.25 \times 10^{21} \mathrm{H}_{2}\right.$ particles/s $)$ and at a pressure of $10^{4} \mathrm{~Pa}$ through a channel of $4 \mathrm{~mm}$ diameter. With an input power of $6.8 \mathrm{~kW}$, a partially ionized and nearly

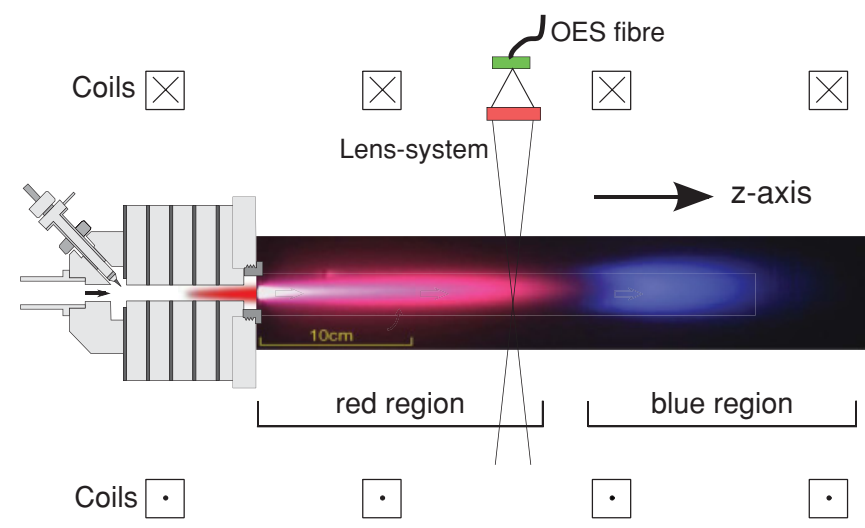

FIG. 2. (Color online) The low-pressure vessel is surrounded by four Helmholtz coils to provide an axially magnetic field of $14 \mathrm{mT}$. The cascaded arc is mounted on a movable arm that allows 3D displacement in the reactor vessel. In this way spatially resolved measurements can be performed while the diagnostic tools remain fixed. The emission of the expanding plasma is dominated by red $\mathrm{H}_{\alpha}$ emission in the first part of the plasma expansion, after which a sharp transition to a blue afterglow is observed. This sharp transition from red to blue occurs at $z=20 \mathrm{~cm}$. fully dissociated hydrogen plasma is produced. It has been shown that in the anode nozzle significant losses of electrons, $\mathrm{H}^{+}$, and $\mathrm{H}$ atoms can occur because of recombination and $\mathrm{H}_{2}{ }^{\text {rv }}$ formation at the surface. Therefore, the shortest nozzle is used since it gives the smallest loss of ions, electrons, and atoms [26].

The plasma expands in a 3-m-long stainless steel cylindrical vessel at low pressure, with a diameter of $30 \mathrm{~cm}$. The pressure gradient between the cascaded arc and the expansion vessel forces the plasma to expand, first supersonically and then, after the formation of a shock, subsonically into the background [27]. The background pressure is typically $9 \mathrm{~Pa}$ in this work. Four Helmholtz coils mounted around the vessel produce a uniform magnetic field of $14 \mathrm{mT}$ over a length of approximately $1.4 \mathrm{~m}$ (see Fig. 2). The created plasma expansion is known to be far from thermal equilibrium [28].

\section{B. Optical emission spectroscopy}

OES is used to detect the visible part of the emission spectra originating from the plasma (see Fig. 2). An optical system consisting of two lenses creates a 1:7 image of the plasma expansion on an optical fiber which guides the collected light to a spectrometer with a spatial resolution of $70 \mu \mathrm{m}$. The latter is based on wavelength dispersion by a Czerny-Turner monochromator and the detection is performed by a light-sensitive CCD detector. A typical spectrum in the blue afterglow is shown in Fig. 3. In the red part of the plasma expansion intensive Fulcher- $\alpha$ band emission $\left(d^{3} \Pi_{u}-a^{3} \Sigma_{g}^{+}\right)$ is observed around $600 \mathrm{~nm}$, indicating higher electron temperatures $\left(T_{e}>1 \mathrm{eV}\right)$ (see Fig. 4). The excited state population is determined by electron (de)excitation processes that depend exponentially on the electron temperature. The total Fulcher- $\alpha$ band emission therefore increases exponentially with the electron temperature.

Absolute calibration of the measured line intensities is achieved with a tungsten ribbon lamp. The intensity of the

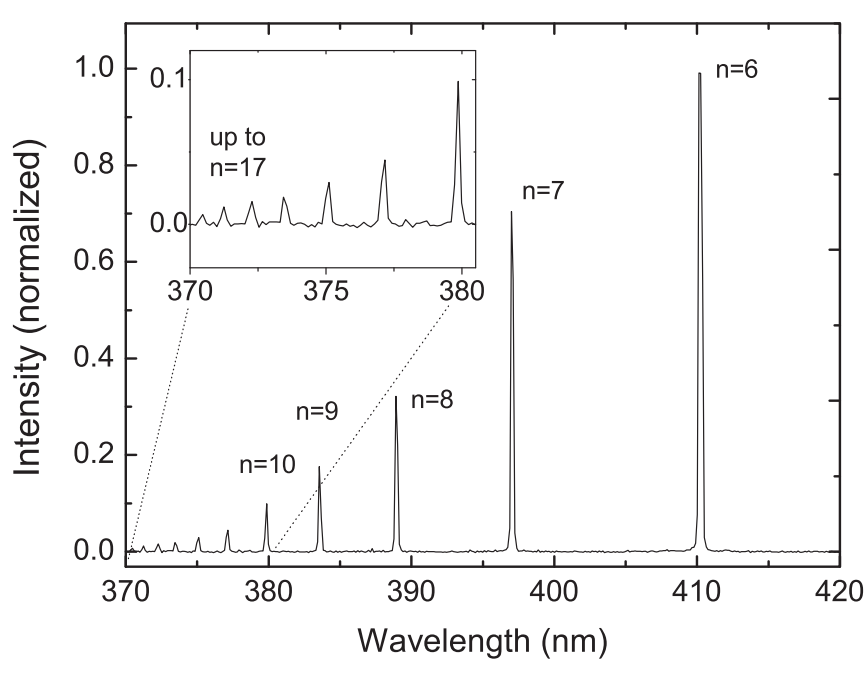

FIG. 3. An emission spectrum obtained with OES in the blue part of the hydrogen plasma expansion at $z=25 \mathrm{~cm}$. The radiation from the lower excited states $n<6$ is not shown here. The experimental conditions are as follows: $I_{\mathrm{arc}}=45 \mathrm{~A}, V_{\mathrm{arc}}=150 \mathrm{~V}$, $\phi_{\mathrm{H}_{2}}=3000 \mathrm{sccm}, p_{B G}=9.3 \mathrm{~Pa}$, and $B_{\mathrm{z}}=14 \mathrm{mT}$. 


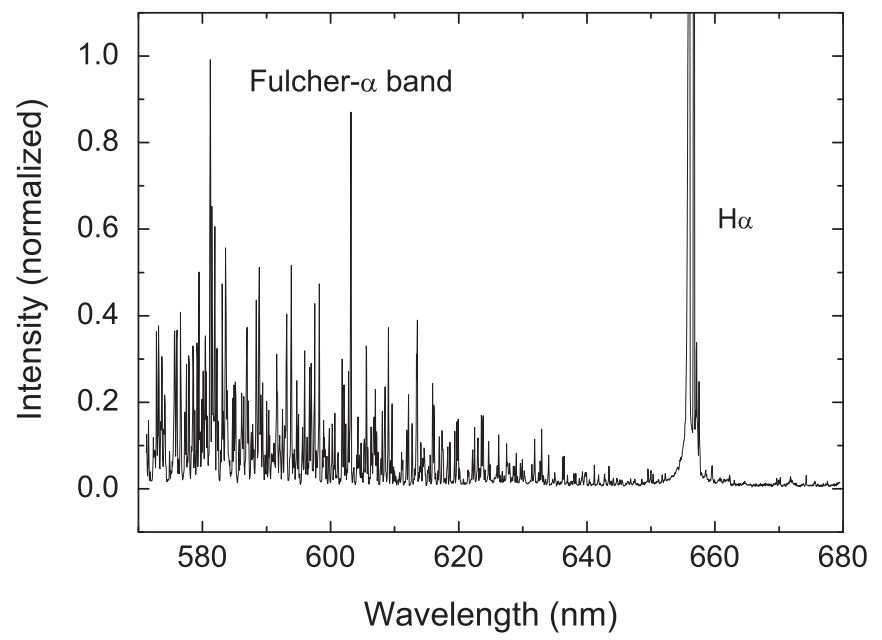

FIG. 4. An emission spectrum obtained with OES in the red part of the hydrogen plasma expansion at $z=4 \mathrm{~cm}$. The emission of the Fulcher band is between 570 and $640 \mathrm{~nm}$, and at $656 \mathrm{~nm} \mathrm{H}_{\alpha}$ emission is observed.

emission is proportional to the density of the upper level $u$. For an optically thin plasma [22], the spectral emission coefficient $j_{\lambda}(\lambda)$ (in $\mathrm{W} \mathrm{m}^{-4} \mathrm{sr}^{-1}$ ) is given by

$$
j_{\lambda}(\lambda)=\frac{1}{4 \pi} n_{u} A_{u l} E_{u l} \varphi_{\lambda}(\lambda)
$$

where labels $u$ and $l$ stand for the upper and lower levels, respectively, $n_{u}$ is the density of the upper level $u, A_{u l}$ (in $\mathrm{s}^{-1}$ ) the Einstein coefficient for spontaneous emission, $E_{u l}$ the energy of the photon that is emitted, and $\varphi_{\lambda}(\lambda)$ the lineshape function of the transition, which is normalized, i.e., $\int \varphi_{\lambda}(\lambda) d \lambda=1$. The spectral radiance $L_{u l}$ along the line-ofsight (LOS) is given as (in $\mathrm{W} \mathrm{m}^{-3} \mathrm{sr}^{-1}$ )

$$
L_{u l}(\lambda)=\int_{\mathrm{LOS}} j_{\lambda}(\lambda) d y=\frac{1}{4 \pi} A_{u l} E_{u l} \varphi_{\lambda}(\lambda) \int_{\mathrm{LOS}} n_{u} d y .
$$

The integration of the spectral radiance over the wavelength gives the radiance $\Lambda_{u l}$ of the line (in $\mathrm{W} \mathrm{m}^{-2} \mathrm{sr}^{-1}$ ):

$$
\Lambda_{u l}=\int L_{u l}(\lambda) d \lambda \equiv \hat{L}_{u l} \Delta \lambda
$$

The $\hat{L}_{u l}$ value can be obtained from an emission measurement and calibrated absolutely with the known spectral density of the tungsten ribbon lamp. The uncertainty of the absolute density calibration with the tungsten ribbon lamp is $10 \%$. The linewidth is $\Delta \lambda$ at full width at half maximum (FWHM) of the Balmer transition. Equation (3) shows that the population density $n_{u}$ of level $u$ depends linearly on $\Lambda_{u l}$,

$$
\hat{n}_{u}=\int_{\mathrm{LOS}} n_{u} d y=\frac{4 \pi \Lambda_{u l}}{A_{u l} E_{u l}}=\hat{L}_{u l} \Delta \lambda \frac{4 \pi}{A_{u l} E_{u l}} .
$$

With the optical setup a lateral profile is measured, i.e., the integrated LOS density. Abel inversion is used to determine the radially resolved densities. The spatial resolution of the optical setup is $3 \mathrm{~mm}$, which is much smaller than the plasma radius. The magnetized plasma expansion has cylindrical symmetry so that Abel inversion of the measured LOS profile can be applied [29]:

$$
n(r)=\frac{-1}{\pi} \int_{r}^{\infty} \frac{\partial \hat{n}\left[\Delta r_{\mathrm{LOS}}(y), y\right]}{\partial y} \frac{1}{\sqrt{y^{2}-r^{2}}} d y .
$$

Here, $\hat{n}\left[\Delta r_{\mathrm{LOS}}(y), y\right]$ is the density along the LOS and $n(r)$ is the spatially resolved density (in $\mathrm{m}^{-3}$ ). The LOS data is analyzed with a purely centralized Gaussian line profile [30]. The observed line profile is a combination of two radial Gaussian profiles, a narrow profile for the plasma core, and a broad profile for the background. A dip in the center of the density profiles can be simulated by adding a third Gaussian with a negative area to the total density profile.

\section{Langmuir double probe}

A Langmuir double probe is used to determine the electron temperature $T_{e}$ and electron density $n_{e}$ in the magnetized expanding hydrogen plasma. The measurement is performed by applying a potential difference between two floating probes and measuring the current passing through the probes to get a symmetrical current-voltage plot [31]. The situation is more complicated when a sufficiently strong magnetic field is applied to the plasma. This will then reduce the electron current collected by the probe. However, the double probe theory is still valid in a magnetized plasma if the Larmor radii for ions and electrons are much larger than the Debye length. Assuming that $n_{e}=10^{18} \mathrm{~m}^{3}, B_{0}=14 \times 10^{-3} \mathrm{~T}$, and $T_{e}=0.3 \mathrm{eV}$, the Larmor radii are $\rho_{i}=3.2 \times 10^{-3} \mathrm{~m}$ and $\rho_{e}=7.0 \times 10^{-5} \mathrm{~m}$ for ions and electrons, respectively. These two are larger than the Debye length $\lambda_{D}=4 \times 10^{-6} \mathrm{~m}$. The influence of the negative ions on the probe theory is small if the density is much lower than the positive ion density [21]. Furthermore, it is also assumed that $\mathrm{H}_{2}{ }^{+}$is dominant over $\mathrm{H}^{+}$ in the blue afterglow due to the importance of the molecular mutual neutralization process. The two probes are both $3.7 \mathrm{~mm}$ long and $0.8 \mathrm{~mm}$ in diameter. The diameter was chosen in such a way that it is much smaller than all relevant mean free path lengths and larger than the Debye length.

It is important to know whether the charged particles in the plasma expansion are magnetized or not. For this the electron cyclotron radius must be smaller than their mean free path in the plasma. The electrons are magnetized once the electron Hall parameter $H_{e i}$ is higher than unity. The electron Hall parameter for the conditions assumed above can be calculated with

$$
H_{e i}=\frac{\lambda_{e i}}{\rho_{e}}=\frac{6.3 \times 10^{22} \times \hat{T}_{e}^{3 / 2} B_{0}}{n_{e} \ln \Lambda} \approx 25 .
$$

This is higher than unity, and therefore the electrons are following the magnetic field lines. The ion Hall parameter can be calculated with

$$
H_{i i}=\frac{\lambda_{i i}}{\rho_{i}}=\frac{2 \times 10^{21} \times \hat{T}_{i}^{3 / 2} B_{0}}{A_{i}^{1 / 2} n_{e} \ln \Lambda} \approx 0.9 .
$$

This shows that the ions are only marginally magnetized. The main conclusion is that the electrons are magnetized, while this is not true for the ions. 
TABLE I. The four input parameters that are obtained in the blue afterglow and used in the atomic CR model.

\begin{tabular}{llc}
\hline \hline Symbol & Obtained via: & Value \\
\hline$T_{e}$ & Double probe & {$[0.9-0.1 \mathrm{eV}]$} \\
$n_{e}$ & Double probe & {$\left[3 \times 10^{18}-2 \times 10^{17} \mathrm{~m}^{-3}\right]$} \\
$T_{\mathrm{gas}}$ & TDLAS & $700 \mathrm{~K}$ \\
$n_{1}$ & TALIF & $10^{20} \mathrm{~m}^{-3}$ \\
\hline \hline
\end{tabular}

\section{ATOMIC COLLISIONAL RADIATIVE MODEL}

A collisional radiative model (CRM) can be used to determine which processes are responsible for the observed population inversion in the blue afterglow. A CRM relates the densities of excited states to those of atomic and ionic ground states. The atomic CRM requires four input parameters to calculate the mass balance for all excited states [32], namely, $T_{e}, n_{e}$, the gas temperature $T_{\mathrm{gas}}$, and the ground state density of atomic hydrogen $n_{1}$. These parameters are derived from experiments conducted on the magnetized hydrogen plasma. The CRM that is used in this work includes three-particle recombination.

In Table I the numerical values of the four input parameters of the CRM are shown. Both the electron density and temperature are derived from the Langmuir double probe measurements. Probe measurements have been performed only for $z>16 \mathrm{~cm}$, as the probe becomes too hot close to the arc. The gas temperature is determined from tunable diode laser absorption spectroscopy (TDLAS) by investigating the Doppler broadening of the $\mathrm{H}_{\alpha}$ absorption signal. The ground state density $n_{1}$ is obtained with two-photon laser-induced fluorescence (TALIF). From the densities of the CR model an atomic state distribution function (ASDF) can be constructed [33]. Knowledge of the ASDF is of fundamental importance since it describes how excited states in atoms and ions are populated at a given electron temperature $T_{e}$, electron density $n_{e}$, and neutral ground-state density $n_{1}$. From the ASDF the coefficients of total recombination and ionization can be calculated which are required for the particle- and energy-source terms in plasma-transport equations.

\section{EXPERIMENTAL RESULTS}

The spatially resolved densities of $n=3$ up to $n=15$, obtained from the Abel inverted OES measurements, are presented. From these data ASDFs are constructed. Subsequently, the densities are compared with the densities obtained from the atomic CRM.

\section{A. Spatially resolved densities}

In Fig. 5(a) the density per statistical weight of $n=3$ is shown $\left(\right.$ in $^{-3}$ ). The background emission at the edges is fitted with a broad Gaussian profile and the center core is fitted with a narrow Gaussian profile. The background emission at the edges is due to metastable species in the recirculating background gas. The local excited state density profiles show that the expansion starts out with a very narrow lateral profile. After the transition, which occurs at $z=18-20 \mathrm{~cm}$, the profile broadens in the blue afterglow.
In Fig. 5(b), the density per statistical weight of $n=4$ is shown. The density of $n=4$ is low in the red part of the plasma expansion where $\mathrm{H}_{\alpha}$ emission is dominant and it increases rapidly after the red-blue transition. An interesting feature is an observed hollow profile in the red-blue transition at $z=18-20 \mathrm{~cm}$.

In Figs. 5(c) and 5(d) we see the density per statistical weight of $n=5$ and 6 , respectively. The densities are low in the red plasma region, and after the red-blue transition we observe that the densities of both $n=5$ and 6 reach a maximum. The hollow profile in the red-blue transition is more pronounced for these higher excited levels (see Fig. 6). From this we conclude that the maximum population inversion is observed toward the edge of the plasma, at $r=1.2 \mathrm{~cm}$, while in the center, at $r=0 \mathrm{~cm}$, no population inversion is observed.

The uncertainties with the Abel inversion method in the red region are expected to be $10 \%$. The uncertainty at the red-to-blue transition that occurs between $z=18$ and $22 \mathrm{~cm}$ for $n=4-6$ is higher and depends on the observed hollow profile. Uncertainties of up to $50 \%$ are expected in the center at $r=0 \mathrm{~mm}$ when the excited state density reaches zero. Abel inversion is well defined in the blue region at distances greater than $z=22 \mathrm{~cm}$ and the uncertainty is not more than $10 \%$.

\section{B. Atomic state distribution function}

From the excited state densities we can construct an ASDF. In Fig. 7(a), an ASDF in the center of the plasma expansion at $z=7 \mathrm{~cm}$ is shown that resembles the typical ASDF of an ionizing plasma of medium electron density [33]. In Fig. 7(b) an ASDF in the blue afterglow, i.e., at $z=33 \mathrm{~cm}$ from the arc exit, is shown. A population inversion of $n=4-6$ with respect to $n=3$ can clearly be detected in the blue afterglow. This shows that there is an input source to the excited states $n=4,5$, and 6 .

\section{The electron temperature and electron density in the plasma}

The electron temperature of the plasma in the cascaded arc was determined to be around $1.5 \mathrm{eV}$ and the electron density $10^{21-22} \mathrm{~m}^{-3}$ [34]. From the Hall parameter it follows that the plasma-charged particles are not magnetized inside the cascaded arc because of the high electron densities.

Since the Langmuir double probe cannot measure close to the arc exit due to thermoemission of the probe, the electron temperature and electron density are unknown in the first $16 \mathrm{~cm}$ of the plasma expansion. Fortunately, the values of $n_{e}$ and $T_{e}$ in the red first part of the expanding plasma column can be determined by observation of the Fulcher system of $\mathrm{H}_{2}{ }^{*}$ and the asymptotic behavior of highly excited states of atomic hydrogen. The highly excited states of atomic hydrogen are shown in Fig. 8.

The presence of significant Fulcher radiation shows that the electron temperature is relatively high in the first part of the expansion. The electron temperature can be determined from the absolute density of the Fulcher band via the following balance:

$$
n_{e} n_{\mathrm{H}_{2}} k^{\mathrm{exc}}=n_{\mathrm{H}_{2}}\left(A_{F}+n_{e} k^{\mathrm{de}-\mathrm{exc}}\right) .
$$



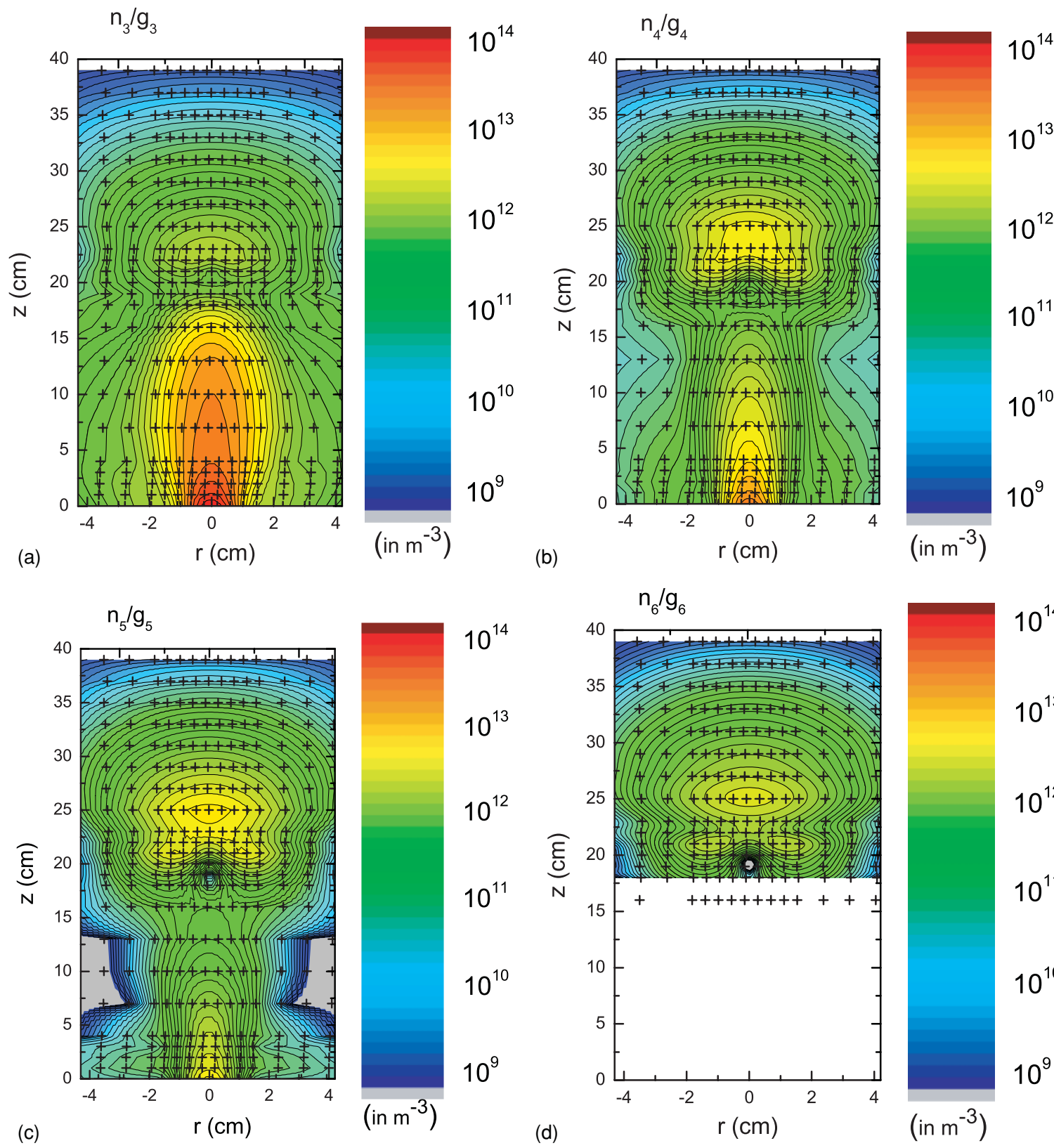

$10^{14}$
$10^{13}$
$10^{12}$
$10^{11}$
$10^{10}$
$10^{9}$
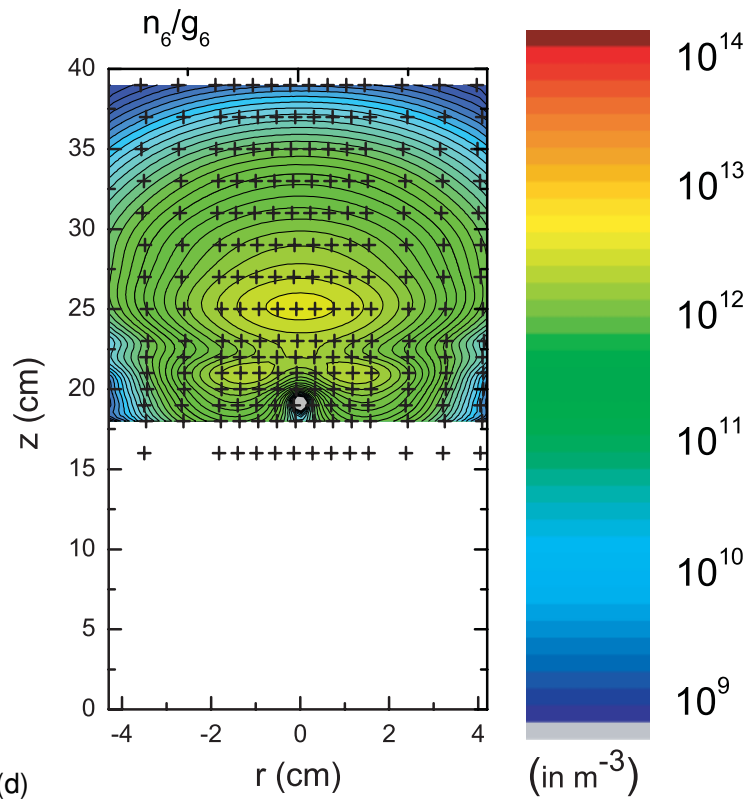

FIG. 5. (Color online) Abel inverted H-atom density profiles divided by the statistical weight (in $\mathrm{m}^{-3}$ ) of $n=3,4,5$, and 6 as a function of $z$, i.e., the axial distance to the exit of the arc.

Here $k^{\text {exc }}=k_{0}^{\text {exc }} \exp \left(-E_{F} / k_{B} T_{e}\right)$ (in $\left.\mathrm{m}^{3} \mathrm{~s}^{-1}\right)$ is the electron excitation rate from the ground state $n_{\mathrm{H}_{2}}\left(X^{1} \Sigma_{g}^{+}\right)$with $k_{0}^{\text {exc }} \simeq 10^{-15} \mathrm{~m}^{3} \mathrm{~s}^{-1}$ [35], $A_{F}=2.5 \times 10^{7} \mathrm{~s}^{-1}$ the transition probability of the Fulcher transition [36], and $k^{\mathrm{de}-\mathrm{exc}} \simeq 1 \times$ $10^{-12} \mathrm{~m}^{3} \mathrm{~s}^{-1}$ the electron de-excitation rate [37]. Furthermore, $n_{\mathrm{H}_{2}}{ }\left(d^{3} \Pi_{u}\right)$ is the upper state density of the Fulcher band emission, and $n_{\mathrm{H}_{2}}$ is the density of molecular hydrogen determined from the ideal gas law. The density of $n_{\mathrm{H}_{2}}$ depends on the gas temperature, which is $3500 \mathrm{~K}$ close to the arc and drops to $1000 \mathrm{~K}$ close to the red-blue transition. The electron temperature can be determined from Eq. (8) with

$$
k T_{e}=\frac{E_{F}}{\ln \left(\frac{n_{\mathrm{H}_{2}} n_{e} k_{0}^{\mathrm{exc}}}{n_{\mathrm{H}_{2} *}\left(A_{F}+n_{e} k^{\mathrm{de}-\mathrm{exc}}\right)}\right)} .
$$

The electron temperatures obtained with Eq. (9) are shown in Fig. 9. Since $n_{e} k^{\mathrm{de}-\mathrm{exc}}>A_{F}$ in the first $16 \mathrm{~cm}$ of the plasma expansion, the electron density drops out.

The electron density in the red part of the plasma expansion can be evaluated by analyzing the excited state distribution (see Fig. 8). The excited states close to the ionization threshold $I_{n}=E_{+}-E_{n} \simeq 0$ are represented by the Saha distribution:

$$
\frac{n_{n}^{S}}{g_{n}}=\frac{n_{e} n_{i}}{g_{e} g_{i}}\left(\frac{h^{2}}{2 \pi m_{e} k_{B} T_{e}}\right)^{3 / 2} \exp \left(\frac{I_{n}}{k_{B} T_{e}}\right),
$$

where $g_{n}$ is the statistical weight of level $n, m_{e}$ the electron mass, $h$ the Planck constant, and $k_{B}$ the Boltzmann constant. From the overpopulation factor $b_{n}=n_{n} / n_{n}^{S}$ [33], which relates the excited state densities with the Saha density, we calculate 


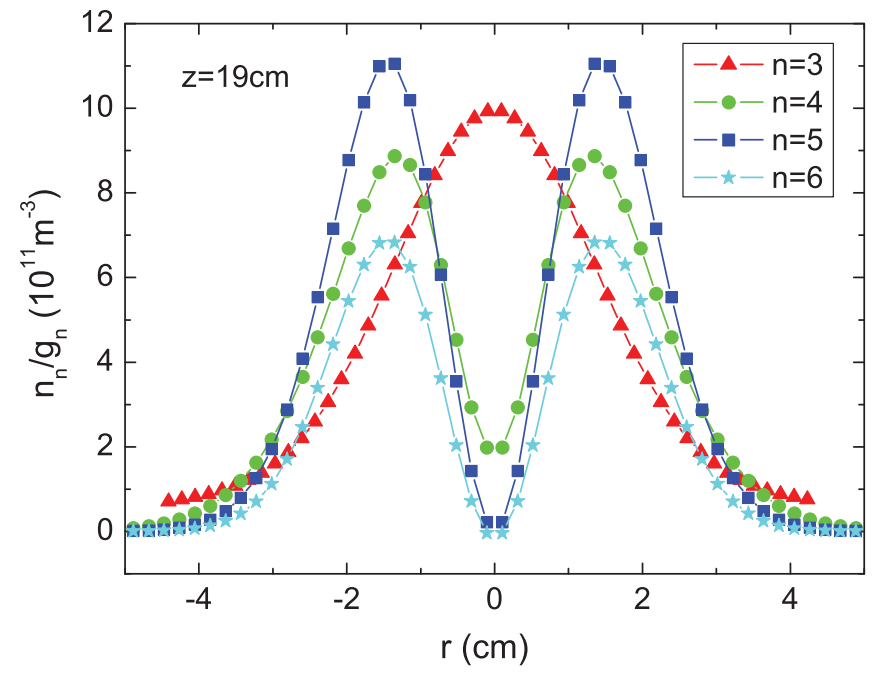

FIG. 6. (Color online) At the red-blue transition, at $z=19 \mathrm{~cm}$, a hollow profile is found for $n=4,5$, and 6 . This clearly shows the creation of highly excited states from species entering the jet from the outside.

that $b_{6-9} \approx 1$ are close to Saha, while the lower excited states are overpopulated with respect to Saha $b_{3-5}>1$. The electron density can be determined from Eq. (10), assuming that $n_{e}=$ $n_{i}, g_{e}=2$, and that the higher excited states are in Saha. The value of $T_{e}$ obtained from the Fulcher band emission is used in these data, since at higher electron densities the overpopulation factor should vary as $b_{n}=1+C T_{e}^{-5.5}$, where $C$ is a constant [33].

Note that the overpopulation factor of the lower Balmer levels is due to MAR processes and only for a smaller amount to direct excitation of $\mathrm{H}$ atoms by electrons. Dissociative excitation of $\mathrm{H}_{2}$ leading to excited $\mathrm{H}$ atom states is here improbable, as this excitation is via a repulsive state and thus requires substantial energy.

In Fig. 9 we observe that the electron density decreases only slightly from $z=17 \mathrm{~cm}$ to $z=24 \mathrm{~cm}$, but decreases significantly for larger $z$ values. The decrease in density starts just in front of the maximum population inversion. With an increasing magnetic field the current out of the cascaded arc extends further into the vessel and thereby increases the electron density. The magnetic field is therefore responsible for shifting the red-to-blue transition and also confining the plasma better.

The electron temperature, measured with the Langmuir double probe, is $0.9 \mathrm{eV}$ at $z=17 \mathrm{~cm}$ and decreases to $0.1 \mathrm{eV}$ at $z=24 \mathrm{~cm}$ from the exit of the arc. After $z=24 \mathrm{~cm}$ the electron temperature remains more or less constant between 0.1 and $0.15 \mathrm{eV}$. Increasing the magnetic field strength does not increase the electron temperature.

The electron temperature determined with the absolute Fulcher band emission and the Langmuir double probe clearly shows a nice overlap between $z=17$ and $19 \mathrm{~cm}$. The comparison of the electron density with the Saha method and the Langmuir double probe shows us that there is overlap as well. The uncertainty in the observation of the Fulcher system
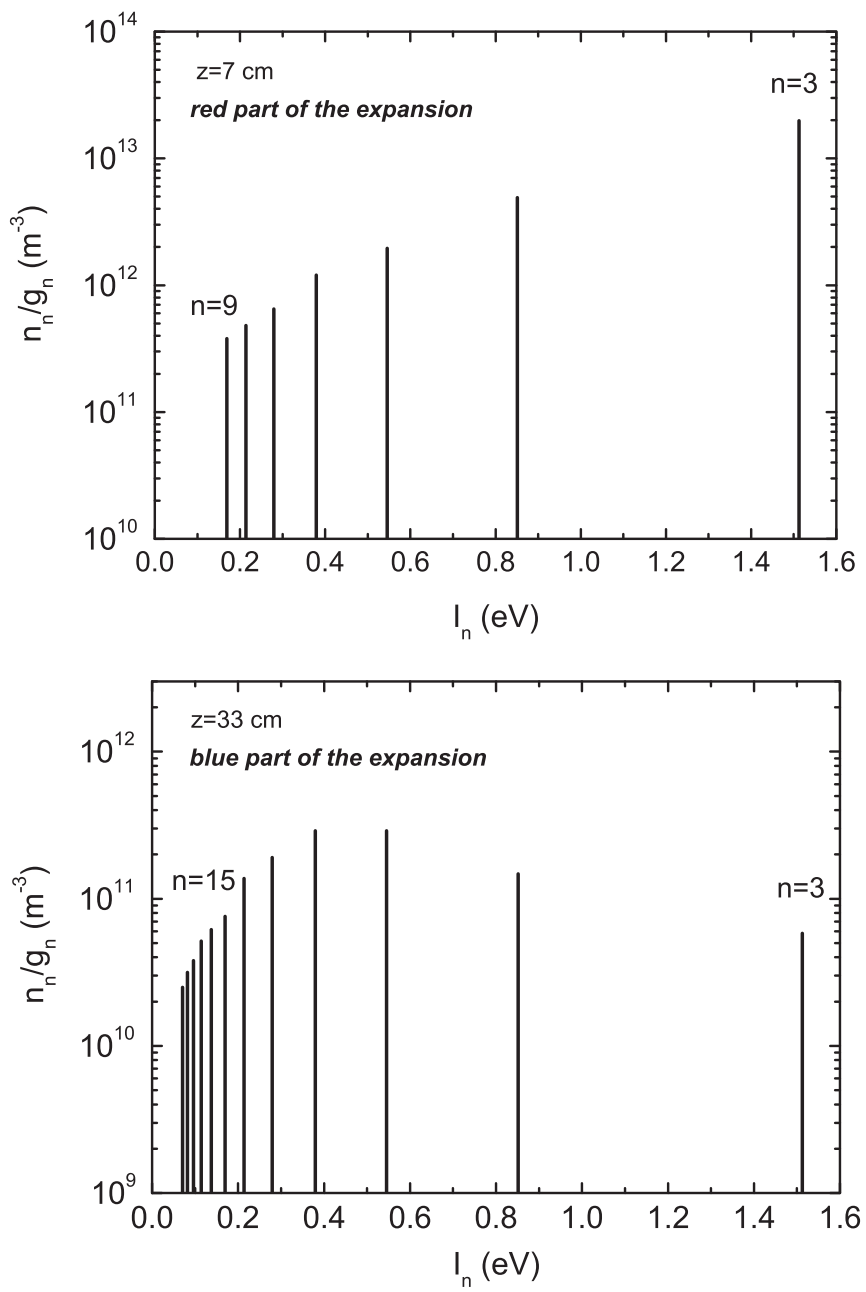

FIG. 7. The atomic hydrogen population density per statistical weight is shown at an axial distance of 7 and $33 \mathrm{~cm}$ from the exit of the arc. Both are measured on-axis at $r=0 \mathrm{~mm}$. In the red region a typical ASDF of an ionizing plasma is observed, and in the blue afterglow population inversion of $n=4,5,6$, and 7 with respect to $n=3$ is observed.

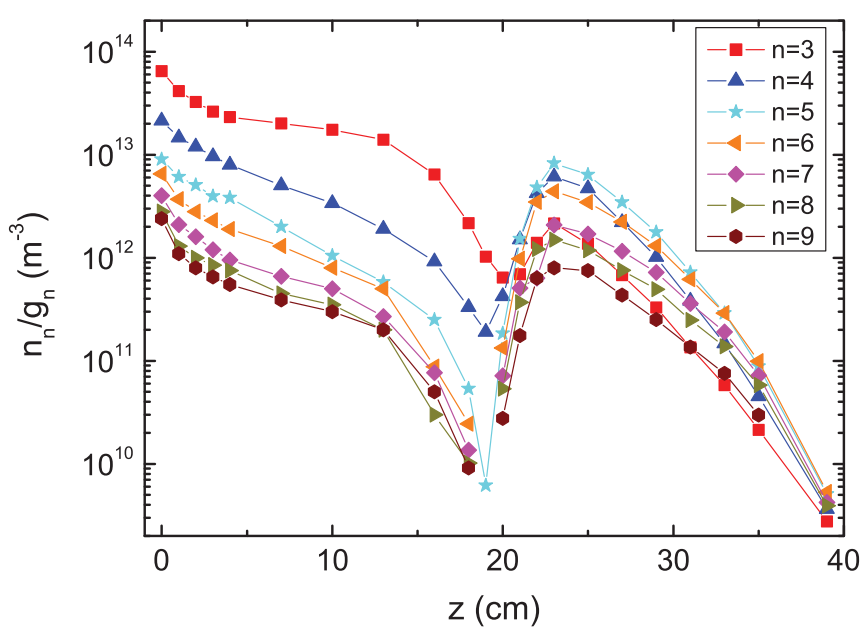

FIG. 8. (Color online) The excited state density as function of the axial position for $n=3$ up to $n=9$ measured on-axis. 


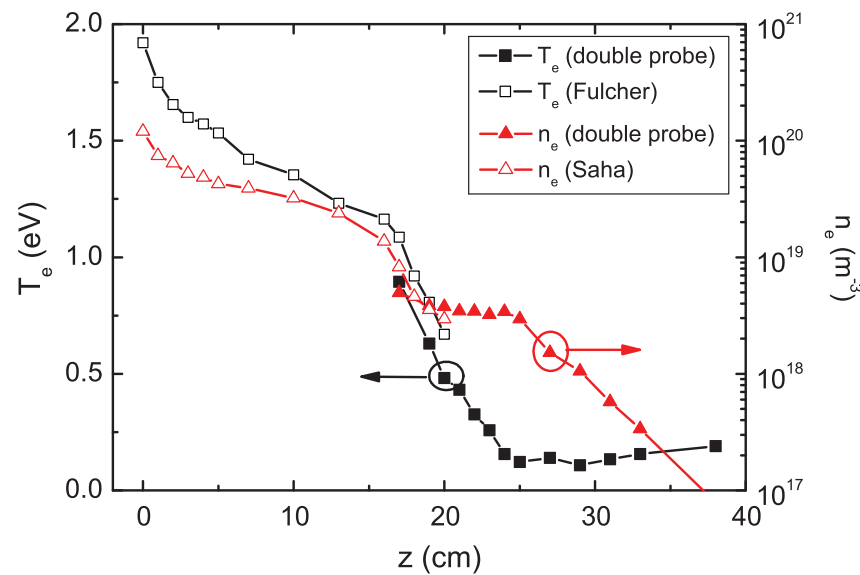

FIG. 9. (Color online) The on-axis electron temperature and electron density determined with the Langmuir double probe and the Saha equilibrium method.

of $\mathrm{H}_{2}{ }^{*}$ and the asymptotic behavior of highly excited states of atomic hydrogen are approximate and estimated to be $30 \%$.

\section{DISCUSSION}

First, the main population processes in the red part of the plasma are outlined. Second, the hollow profile in the red-blue transition is discussed followed by the main processes in the blue afterglow.

\section{A. The red plasma region}

As shown in Fig. 5, the densities of excited states is highest measured for $n=3$ in the red part of the plasma expansion. The dominant chemical process in the red plasma expansion to produce excited atoms is MAR. The creation of this reaction is done in two steps. First, a molecular ion is produced by charge exchange with a proton [38]:

$$
\mathrm{H}_{2}{ }^{\mathrm{rv}}+\mathrm{H}^{+} \rightarrow \mathrm{H}_{2}{ }^{+, \mathrm{rv}}+\mathrm{H}(n=1) \text {. }
$$

In this step, the hydrogen molecule needs to be ro-vibrationally excited since this reaction is otherwise endothermic with an energy deficit of $2.1 \mathrm{eV}$. The second step is dissociative recombination of $\mathrm{H}_{2}{ }^{+, \mathrm{rv}}$ in which two hydrogen atoms are produced, i.e., one in the ground state and another in an excited state:

$$
\mathrm{H}_{2}{ }^{+, \mathrm{rv}}+e \rightarrow \mathrm{H}(n=1)+\mathrm{H}(n=2,3) .
$$

Notably, to produce an excited hydrogen atom in a state with $n \geqslant 4$, the ro-vibrationally excited hydrogen molecule should have an internal energy of at least $3.7 \mathrm{eV}$. Vankan et al. [7] showed with vacuum ultraviolet laser induced fluorescence (VUV-LIF) experiments that the internal energy of $\mathrm{H}_{2}{ }^{\text {rv }}$ close to the arc is not much higher than $2.5 \mathrm{eV}$. This shows that dissociative recombination is only important in the red part of the plasma expansion populating $n=2$ and 3 . The population of the higher excited states $n=4$ and 5 can be formed by collisional excitation from $n=3$ due to the high electron temperature $\left(T_{e}>1 \mathrm{eV}\right)$.

\section{B. The red-blue transition}

The hollow profile obtained in the red-blue transition at $z=18-22 \mathrm{~cm}$ suggests that there is an inward flow of $\mathrm{H}_{2}{ }^{\mathrm{rv}}$ from the vessel wall, producing $\mathrm{H}^{-}$ions [39]. Since the positive ions and electrons are mostly confined in the center of the plasma and the ro-vibrationally excited molecules are more dense away from the center axis of the plasma, an optimum of the dissociative attachment process occurs. Hence, the negative ions form a hollow profile and react with the positive molecular ions via the molecular mutual neutralization process to form the highly excited states that are observed. The importance of charged particles at the red-to-blue transition is shown by the influence of the magnetic field on the transition. With an increasing magnetic field the current out of the cascaded arc extends further into the vessel and thereby increases the electron density. The magnetic field is therefore responsible for shifting the red-to-blue transition and also confining the plasma better. Increasing the magnetic field strength does not increase the electron temperature significantly. These results are also confirmed with Thomson scattering by Shumack et al. [40]. The magnetic field dependence shows that the transition cannot be explained by flow patterns, e.g., recirculation cells, because the ionization degree of the plasma is too low to alter the flow because of the presence of charged particles.

\section{The blue afterglow}

Because of the low electron temperature and relatively high electron density in the blue region, three-particle recombination, i.e., $\mathrm{H}^{+}+e+e \rightarrow \mathrm{H}(n)+e$, can become significant on the center axis, i.e., $r=0 \mathrm{~mm}$. To investigate the importance of three-particle recombination on the density of the excited states, an ASDF is constructed from the results of the CRM (see Fig. 10). The ASDF at $z=33 \mathrm{~cm}$ shows that the CR model predicts the densities to be more than two orders of

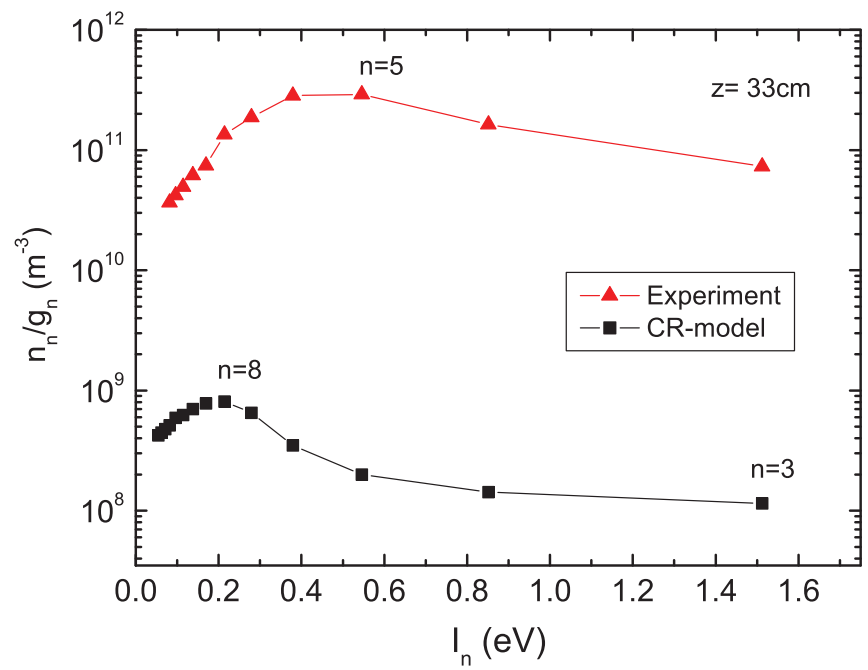

FIG. 10. (Color online) Comparison of the atomic CRM with the data retrieved from OES at $z=33 \mathrm{~cm}$. The densities with the model are more than two orders of magnitude lower than the OES measurements, and the maximum population is at a higher quantum number, $n=8$ instead of $n=5$. Note that the measurement is done on-axis $(r=0 \mathrm{~mm})$ at $z=33 \mathrm{~cm}$. 
magnitude lower than measured. The reason that three-body recombination cannot explain the high densities of the excited states is because the electron densities in the plasma are too low. To discuss the importance of three-body recombination in the blue afterglow the mass balance is used:

$$
n_{e}^{3} k^{\mathrm{rec}}=\frac{n_{4}}{\tau_{4}}
$$

Here, $k^{\text {rec }}$ is the three-body recombination coefficient given by $k^{\mathrm{rec}}=1.7 \times 10^{-39} \times T_{e}^{-9 / 2} \mathrm{~m}^{6} \mathrm{~s}^{-1}$ with $T_{e}$ in eV and $\tau_{4}$ the lifetime of state $n=4$. Since $\tau_{4}^{-1}=A_{4}=3 \times 10^{-7} \mathrm{~s}^{-1}$, $n_{e}=3.4 \times 10^{17} \mathrm{~m}^{-3}$, and $T_{e}=0.16 \mathrm{eV}$, the maximum value at $z=33 \mathrm{~cm}$ of $n_{4}$ is $2 \times 10^{9} \mathrm{~m}^{-3}$. The value measured with OES is two orders of magnitude higher than obtained with the atomic CRM. This makes it very improbable that only atomic processes are responsible for the measured high densities in the magnetized hydrogen plasma. Therefore, molecular processes must be dominant for the formation of the measured excited states. Furthermore, the production rate of each excited state [19] correlates very well with the shape of the cross section of $\mathrm{H}_{2}{ }^{+}+\mathrm{H}^{-}$[24]. To study the importance of these molecular processes a more advanced $\mathrm{CR}$ model is needed in which molecular processes are included [22,41]. This is necessary to prove that the observed population inversion in the blue afterglow is caused by MAR processes.

\section{CONCLUSION}

The magnetized hydrogen plasma expansion shows a typical red region in the first $20 \mathrm{~cm}$ of the expansion, after which a sharp transition to a blue afterglow is observed. The Balmer emission lines of the plasma are investigated with optical emission spectroscopy to determine the absolute densities of electronically excited hydrogen states from $n=3$ up to $n=15$. From these measurements we observed population inversion of $n=4-6$ with respect to $n=3$ in the blue afterglow. The electron temperature in the red part of the plasma is determined from the absolute Fulcher band emission and the electron density from the Saha method by looking at the excited state densities that are close to Saha. In conjunction with the Langmuir double probe measurements, a complete picture of $n_{e}$ and $T_{e}$ is given in the plasma on-axis.

An important part of this paper is devoted to the discussion of which population mechanisms are responsible for the population inversion in the blue afterglow. The two most likely population mechanisms proposed for the population inversion in the blue afterglow are three-body recombination of $\mathrm{H}^{+}$with two electrons and molecular mutual neutralization of $\mathrm{H}_{2}{ }^{+}$with $\mathrm{H}^{-}$. The importance of the latter process is shown at the red-blue transition where a hollow profile of the excited states $n=4-6$ is observed. The hollow profile could indicate a lateral inflow of $\mathrm{H}_{2}{ }^{\mathrm{rv}}$, produced at the reactor walls. This can lead to an increase in asymmetric charge exchange and therefore tip the balance between molecular and atomic recombination. The position of the red-to-blue transition is, however, strongly influenced by the magnetic field. This excludes the possibility that the transition is purely caused by flow patterns like recirculation cells. The magnetic field dependence means that the electron density and temperature play an important role in the transition.

Comparing the measured densities with the densities calculated on the basis of the measured $T_{e}$ and $n_{e}$, using an atomic collisional radiative model, leads to the conclusion that atomic recombination processes cannot account for the large population densities observed. Therefore, it is concluded that MAR processes are responsible for the population inversion in the blue afterglow.

\section{ACKNOWLEDGMENTS}

We greatly appreciate the skillful technical assistance of M. J. F. van de Sande, J. J. A. Zeebregts, J. J. L. M. Meulendijks, and H. M. M. de Jong. This work is part of the research program of the Dutch Foundation for Fundamental Research on Matter (FOM-TFF). It is also supported by the European Communities under the contract of Association between EURATOM and FOM and carried out within the framework of the European Fusion Programme.
[1] M. N. van den Donker, B. Rech, and F. Finger, Appl. Phys. Lett. 87, 263503 (2005).

[2] W. M. M. Kessels, R. J. Severens, A. H. M. Smets, B. A. Korevaar, G. J. Adriaenssens, D. C. Schram, and M. C. M. van de Sanden, J. Appl. Phys. 89, 2404 (2001).

[3] J. D. Sherman and Y. J. Belchenko, AIP Conf. Proc. 763 (2004).

[4] J. G. Alessi and A. Hershcovitch, AIP Conf. Proc. 387 (1994).

[5] M. J. de Graaf, R. Severens, R. M. Dahiya, M. C. M. van de Sanden, and D. C. Schram, Phys. Rev. E 48, 2098 (1993).

[6] N. Ohno, N. Ezumi, S. Takamura, S.I. Krasheninnikov, and A.Y. Pigarov, Phys. Rev. Lett. 81, 818 (1998).

[7] P. Vankan, D. C. Schram, and R. Engeln, Chem. Phys. Lett. 400, 9876 (2004).

[8] D. E. Post, J. Nucl. Mater. 220, 143 (1995).

[9] S. I. Krasheninnikov, A. Y. Pigarov, D. A. Knoll, B. LaBombard, B. Lipschultz, D. J. Sigmar, K. J. Soboleva, J. L. Terry, and F. Wising, Phys. Plasmas 4, 1638 (1997).

[10] S. I. Krasheninnikov, A. Y. Pigarov, and D. J. Sigmar, Phys. Lett. A 314, 285 (1996).
[11] A. Y. Pigarov and S. I. Krasheninnikov, Phys. Lett. A 222, 251 (1996).

[12] H. Maecker, Z. Naturf. 11, 457 (1956).

[13] G. M. W. Kroesen, D. C. Schram, A. T. M. Wilbers, and G. J. Meeusen, Contrib. Plasma Phys. 31, 27 (1991).

[14] M. C. M. van de Sanden, J. M. Regt, and D. C. Schram, Phys. Rev. E 47, 2792 (1993).

[15] M. C. M. van de Sanden, J. M. Regt, and D. C. Schram, Plasma Sources Sci. Technol. 3, 501 (1994).

[16] R. F. G. Meulenbroeks, R. Engeln, M. N. A. Beurskens, R. M. J. Paffen, M. C. M. van de Sanden, J. A. M. van der Mullen, and D. C. Schram, Plasma Sources Sci. Technol. 4, 74 (1995).

[17] L. I. Gudzenko and L. A. Shelepin, Zh. Eksp. Teor. Fiz. 45, 1445 (1963).

[18] H. Akatsuka and M. Susuki, Phys. Rev. E 49, 2 (1994).

[19] J. J. A. van den Dungen, O. Gabriel, H. S. M. M. Elhamali, D. C. Schram, and R. Engeln, IEEE Trans. Plasma Sci. 36, 1028 (2009).

[20] H. Akatsuka and M. Suzuki, Phys. Rev. E 49, 1534 (1993). 
[21] K. Shibagaki and K. Sasaki, J. Phys. D: Appl. Phys. 41, 195204 (2008).

[22] Z. Qing, D. K. Otorbaev, G. J. H. Brussaard, M. C. M. van de Sanden, and D. C. Schram, J. Appl. Phys. 80, 1312 (1996).

[23] R. F. G. Meulenbroeks, M. C. M. van de Sanden, and D. C. Schram, Phys. Rev. Lett. 82, 10 (1999).

[24] M. J. J. Eerden, M. C. M. van de Sanden, D. K. Otorbaev, and D. C. Schram, Phys. Rev. A 51, 3362 (1995).

[25] O. G. Gabriel, D. C. Schram, and R. A. H. Engeln, Phys. Rev. E 78, 016407 (2008).

[26] O. G. Gabriel, D. C. Schram, and R. Engeln, Phys. Rev. E 78, 1 (2008).

[27] S. Mazouffre, M. G. H. Boogaarts, I. S. J. Bakker, P. Vankan, R. Engeln, and D. C. Schram, Phys. Rev. E 64, 016411 (2001).

[28] V. M. Lelevkin, D. K. Otorbaev, and D. C. Schram, Physics of Non-Equilibrium Plasmas (Elsevier, 1992).

[29] T. Chongli and Z. Yan, Plasma Sci. Technol 8, 5 (2006).

[30] O. Bendinelli, L. Ciotti, and G. Parmeggiani, Astron. Astrophys. 279, 668 (1993).

[31] G. J. H. Brussaard, M. van der Steen, M. Carrèere, M. C. M. van de Sanden, and D. C. Schram, Phys. Rev. E 54, 1906 (1996).
[32] A. Hartgers, J. van Dijk, J. Jonkers, and J. A. M. van der Mullen, Comput. Phys. Commun. 135, 2 (2001).

[33] J. A. M. van der Mullen, Phys. Rep. 191, 109 (1990).

[34] J. J. Beulens, M. J. de Graaf, G. M. W. Kroesen, and D. C. Schram, Mat. Res. Symp. Proc. 190, 311 (1991).

[35] R. Riahi, P. Teulet, Z. Ben Lakhdar, and A. Gleizes, Eur. Phys. J. D 56, 61 (2010).

[36] G. R. Mohlmann and F. J. de Heer, Chem. Phys. Lett. 43, 240 (1976).

[37] C. Park, J. Quant. Spectrosc. Radiat. Transf. 1, 7 (1970).

[38] D. C. Schram, R. A. B. Zijlmans, O. Gabriel, and R. Engeln, J. Phys.: Conf. Ser. 192, 012012 (2009).

[39] R. F. G. Meulenbroeks, D. C. Schram, M. C. M. van de Sanden, and J. A. M. van der Mullen, Phys. Rev. Lett. 76, 1840 (1996).

[40] A. E. Shumack, V. P. Veremiyenko, D. C. Schram, H. J. de Blank, W. J. Goedheer, H. J. van der Meiden, W. A. J. Vijvers, J. Westerhout, N. J. Lopes Cardozo, and G. J. van Rooij, Phys. Rev. E 78, 11 (2008).

[41] U. Fantz and D. Wunderlich, New J. Phys. 8, 301 (2006). 